\title{
Branched-chain amino acid catabolism is a conserved regulator of physiological ageing
}

\author{
Johannes Mansfeld 1 ,2,3,4, Nadine Urban ${ }^{3, \dagger}$, Steffen Priebe $4,5,6$, Marco Groth ${ }^{4,7}$, Christiane Frahm ${ }^{4,8}$, \\ Nils Hartmann ${ }^{4,9}$, Juliane Gebauer ${ }^{4,10}$, Meenakshi Ravichandran ${ }^{1}$, Anne Dommaschk ${ }^{3, \dagger}$, Sebastian Schmeisser ${ }^{3, \dagger}$, \\ Doreen Kuhlow $3,4,11$, Shamci Monajembashi2 ${ }^{12}$ Sibylle Bremer-Streck ${ }^{13}$, Peter Hemmerich ${ }^{4,12}$, \\ Michael Kiehntopf ${ }^{13}$, Nicola Zamboni ${ }^{14}$, Christoph Englert ${ }^{4,9,15}$, Reinhard Guthke ${ }^{4,6}$, Christoph Kaleta ${ }^{4,10,15, \dagger}$,

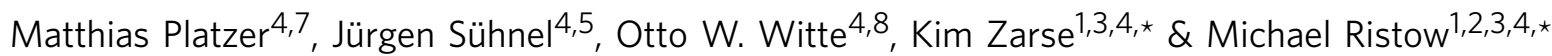

Ageing has been defined as a global decline in physiological function depending on both environmental and genetic factors. Here we identify gene transcripts that are similarly regulated during physiological ageing in nematodes, zebrafish and mice. We observe the strongest extension of lifespan when impairing expression of the branched-chain amino acid transferase-1 (bcat-1) gene in C. elegans, which leads to excessive levels of branched-chain amino acids (BCAAs). We further show that BCAAs reduce a LET-363/mTOR-dependent neuro-endocrine signal, which we identify as DAF-7/TGF $\beta$, and that impacts lifespan depending on its related receptors, DAF- 1 and DAF-4, as well as ultimately on DAF-16/FoxO and HSF-1 in a cell-non-autonomous manner. The transcription factor HLH-15 controls and epistatically synergizes with BCAT-1 to modulate physiological ageing. Lastly and consistent with previous findings in rodents, nutritional supplementation of BCAAs extends nematodal lifespan. Taken together, BCAAs act as periphery-derived metabokines that induce a central neuro-endocrine response, culminating in extended healthspan.

\footnotetext{
${ }^{1}$ Energy Metabolism Laboratory, Swiss Federal Institute of Technology (ETH) Zurich, CH-8603 Zurich, Switzerland. ${ }^{2}$ DFG Graduate School of Adaptive Stress Response \#1715, D-07745 Jena, Germany. ${ }^{3}$ Department of Human Nutrition, Institute of Nutrition, Friedrich-Schiller-University Jena, D-07743 Jena, Germany. ${ }^{4}$ GerontoSysJenAge Consortium, BMBF 0315581, D-07745 Jena, Germany. ${ }^{5}$ Biocomputing Group, Leibniz Institute on Aging-Fritz Lipmann Institute, D-07745 Jena, Germany. ${ }^{6}$ Systems Biology and Bioinformatics Group, Leibniz Institute for Natural Product Research and Infection Biology, HansKnöll-Institute, D-07745 Jena, Germany. ${ }^{7}$ Genome Analysis, Leibniz Institute on Aging-Fritz Lipmann Institute, D-07745 Jena, Germany. ${ }^{8}$ Hans Berger Department of Neurology, Jena University Hospital, D-07747 Jena, Germany. ${ }^{9}$ Molecular Genetics, Leibniz Institute on Aging-Fritz Lipmann Institute, D07745 Jena, Germany. ${ }^{10}$ Research Group Theoretical Systems Biology, Friedrich-Schiller-University Jena, D-07743 Jena, Germany. ${ }^{11}$ German Institute of Human Nutrition Potsdam-Rehbrücke, D-14558 Nuthetal, Germany. ${ }^{12}$ Imaging Facility, Leibniz Institute on Aging - Fritz Lipmann Institute, D-07745 Jena, Germany. ${ }^{13}$ Institute of Clinical Chemistry and Laboratory Medicine, University of Jena, D-07743 Jena, Germany. ${ }^{14}$ Institute of Molecular Systems Biology, Swiss Federal Institute of Technology (ETH) Zurich, CH-8093 Zürich, Switzerland. ${ }^{15}$ Faculty of Biology and Pharmacy, Friedrich-Schiller-University Jena, 07743 Jena, Germany. † Present addresses: Department of Nutrigenomics, Institute of Nutrition, Friedrich-Schiller-University Jena, D-07743 Jena, Germany (N.U.); Department of Respiratory Medicine, Hannover Medical School, D-30625 Hannover, Germany (A.D.); Department of Biology, McGill University, Montréal, Quebec, Canada H3A OG4 (S.S.); Research Group Medical Systems Biology, Institute of Experimental Medicine, Christian-Albrechts-University Kiel, D-24105 Kiel, Germany (C.K.). * These authors contributed equally to this work. Correspondence and requests for materials should be addressed to M.R. (email: michael-ristow@ethz.ch).
} 
W hile the process of ageing has been fascinated humankind for several thousands of years, interventions that reproducibly delay physical decline have first been described less than a century ago ${ }^{1,2}$. With the rise of genetic methods, specific pathways that control the process of ageing have been identified and analysed, giving rise to novel approaches to improve quality of life at higher age ${ }^{3,4}$.

The first systematic studies to identify random mutations associated with longevity were performed in the model organisms Caenorhabditis elegans ${ }^{2}$. Subsequently, other organisms including Saccharomyces cerevisiae, Drosophila melanogaster, rodents and more recently several fish models were employed to dissect genetic pathways linked to physiological ageing. The best-studied one is the insulin/IGF-1 signalling pathway that has been identified in C. elegans to extend lifespan ${ }^{5,6}$. This role has then been extended to other organisms including Drosophila ${ }^{7,8}$ and rodents ${ }^{9-11}$. Given its evidently conserved nature, it was anticipated and subsequently confirmed that this pathway may impact human longevity as well ${ }^{12-14}$. While this and few other signalling pathways mediated by AMP-activated protein kinase $(\mathrm{AMPK})^{15}$, mammalian target of rapamycin $(\mathrm{mTOR})^{16,17}$, sirtuins ${ }^{18}$ or reactive oxygen species ${ }^{19,20}$ are evolutionary conserved, multiple others appear restricted to individual model organisms with little or no impact to mammals nor humans.

Concomitantly, unbiased screening approaches to identify ageing-associated genes and pathways have been performed for single model organisms indicating that $\sim 1$ per cent of coding genes may impact the lifespan of nematodes ${ }^{21,22}$. Subsequently and to identify conserved pathways that may apply to several species, two invertebrate model organisms have been compared ${ }^{23}$, and genes identified in S. cerevisiae have been, at least in part, found to be conserved in C. elegans ${ }^{24}$.

We here have extended these approaches by comparing ageingrelated gene expression patterns in three different organisms, namely $C$. elegans, zebrafish and mice, to identify ageing-related regulations of gene expression levels. We identify a crucial step for the catabolism of branched-chain amino acids (BCAAs), encoded by a gene named branched-chain amino acid transferase 1 (bcat-1) to be consistently regulated in three different organisms, and dissect the signalling role of BCAAs in C. elegans to promote increased healthspan.

\section{Results}

Identification of ageing-related genes in three species. We have studied three well-established model organisms, the invertebrate nematode C. elegans, the vertebrate fish Danio rerio and the mammalian Mus musculus strain C57BL/6J, to identify genes that are similarly regulated on a transcriptional level during physiological ageing. We obtained skin samples from individual zebrafish and mice, as well as pellets containing $\sim 2,000$ C. elegans Bristol N2, at three different ages (see Fig. 1 for details). RNA was extracted from these samples and subjected to Illumina next-generation sequencing (RNA-seq). About 13-82 million reads were obtained for each individual sample (Supplementary Data 1-3). Data analysis had to be restricted to genes for which orthologs could be identified in all three species (C. elegans: 4,850; D. rerio: 6,064; M. musculus: 5,904; Fig. 1). Those with transcript levels showing statistically significant differences by both DESeq and edgeR at least between two time points or by the baySeq test over the three time points were regarded as differentially expressed genes (DEG; C. elegans: 3,608; D. rerio: 1,721; M. musculus: 339; Supplementary Data 1-3). All DEGs were combined and the expression profiles optimally clustered into six courses, two of which each showed global up- or downregulation

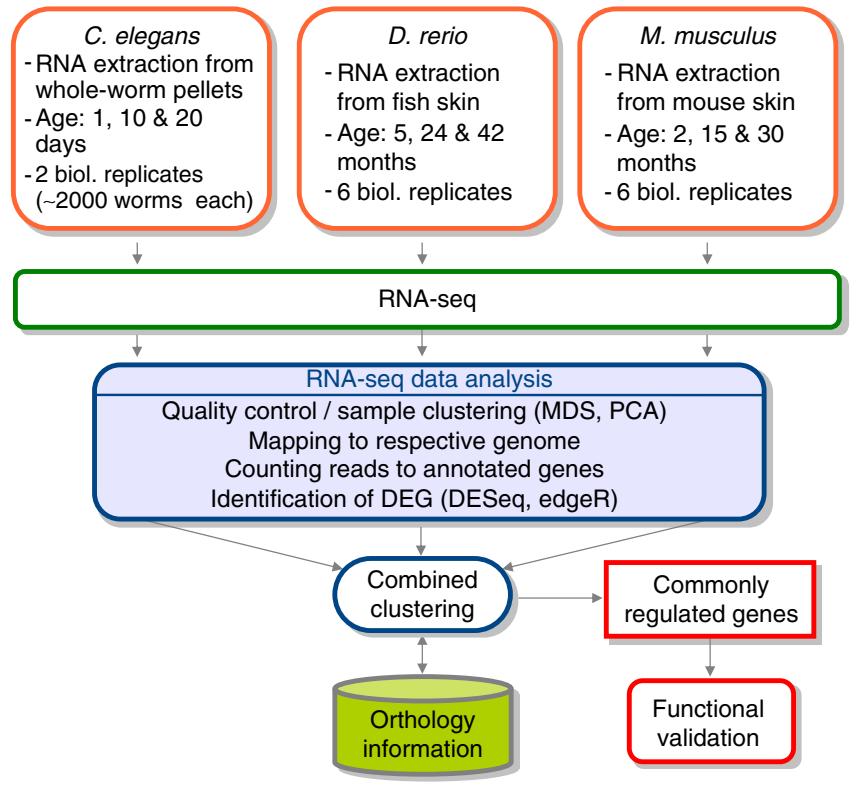

Figure 1 | Sample acquisition and data processing scheme of the transspecies screening approach. RNAs of each sample were sequenced. After passing quality control and sample clustering, the sequences were mapped to the referring genome. The number of reads of the resulting annotated genes were used for statistical evaluation. Commonly regulated genes over the three species were subsequently tested individually for putative impact on lifespan in C. elegans.

with ageing, respectively (Fig. 2 and Supplementary Fig. 1). We identified 13 genes to be upregulated during ageing in all three species, while 16 genes were found to be downregulated (Fig. 2).

RNAi-based validation of ageing-related genes in C. elegans. All of these 29 genes and 12 additionally predicted paralogs (Supplementary Table 1) were individually targeted by feeding respective RNA interference (RNAi) to young adult worms, revealing that 30 out of 41 genes $(73 \%)$ have an individual effect on life expectancy in worms (Table 1, Figs 3-6 and Supplementary Table 2).

Out of these 41 genes, interference with 12 genes did extend mean lifespan by $5 \%$ or more (Table 1 and Supplementary Table 2). The most pronounced extension of lifespan was observed when applying RNAi against a crucial step for the catabolism of BCAAs, encoded by a gene named bcat-1.

Impaired bcat-1 expression extends $C$. elegans lifespan. Applying RNAi against bcat-1 did abolish expression of the gene (Fig. 7a) and extended mean lifespan by $25 \%$ and maximum lifespan by $19 \%$, reflecting the strongest effect of all genes identified (Fig. 7b, Figs 3-6 and Supplementary Table 2). We next reanalysed our RNA samples using quantitative PCR (qPCR) to validate the RNA-seq results, and confirmed the downregulation of bcat-1 transcript levels during physiological ageing in all three species (Fig. 7c). Additional support for the important role of BCAT-1 during ageing comes from an analysis of differentially regulated metabolic pathways during ageing in C. elegans, in which we found that the degradation of BCAAs, whose first step is catalysed by BCAT-1, was the most significantly downregulated pathway (26 out of 29 BCAA-metabolizing reaction steps, Supplementary Table 3). Moreover, published metabolomicsbased evidence suggest that BCAAs are upregulated in long-lived daf-2 nematodes in a daf-16-dependent manner 'making them 
strong candidates for being causally involved in longevity' ${ }^{25}$ while a putative role of bcat-1 has not been analysed in this regard. Taken together these findings suggest that bcat-1 expression may promote ageing, and that ageing organisms may endogenously downregulate this gene, potentially to counteract ageing across species.

We next applied RNA-seq to nematodes exposed to bcat-1 RNAi for 5 days, and analysed the number of genes consistently regulated during physiological ageing (Fig. 2) as well as in the state of bcat-1 impairment. We found that physiological ageing and bcat-1 impairment affects DEGs similarly where $45.8 \%$ (physiological ageing) and 54\% (bcat-1 RNAi) of the respective DEGs overlapped (Fig. 7d). Moreover, we found that physiological ageing and bcat-1 RNAi up- or downregulate the same DEGs, respectively: while 1700 DEGs were up- and 1378 DEGs were downregulated in the same direction, only 619 and

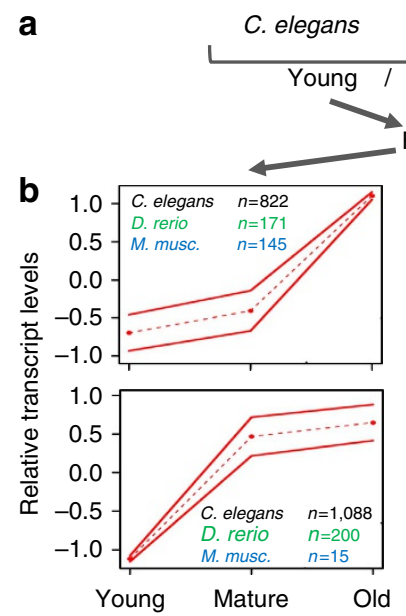

D. rerio M. musculus

d

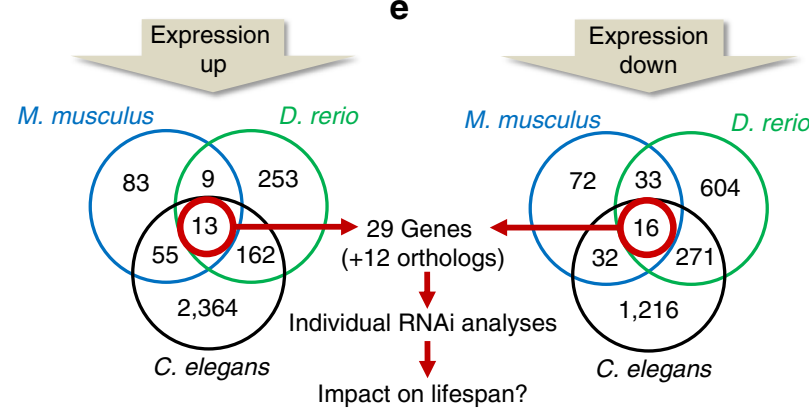

Figure 2 | A trans-species screening approach to identify ageing-

associated genes. (a) Depicts species subjected to RNA extraction at three different ages. (b) Depicts relative RNA transcript levels uniformly upregulated during physiological ageing. (c) Depicts relative RNA transcript levels uniformly downregulated during physiological ageing. (d,e) Show results of Venn analysis from genes identified in $\mathbf{b}$ and $\mathbf{c}$, respectively. C. elegans results are shown in black, D. rerio in green and M. musculus in blue.
822 DEGs were regulated in an opposing manner (Fig. 7e), respectively. Lastly, statistical analysis revealed a highly significant (MonteCarlo, $P=0.00099$ ) correlation between DEGs of normal ageing versus bcat-1 RNAi, suggesting a global functional relevance of BCAT-1 for the process of physiological ageing.

Impairing bcat-1 expression promotes $C$. elegans healthspan. We next aimed at quantifying a number of ageing-associated parameters, including the accumulation of previously established ageing pigments ${ }^{26}$, which was observed to be reduced in 13-daysold nematodes exposed to bcat-1 RNAi (Fig. 7f). While interventions to extend lifespan typically reduce fecundity, we did not observe such effect of bcat-1 RNAI treatment (Fig. $7 \mathrm{~g}$ and Supplementary Fig. 2a). In addition, we quantified maximum movement speed of nematodes and found impairment of bcat-1 expression to increase this parameter (Fig. 7h). Hence, reduced bcat-1 expression extends lifespan without affecting fecundity, reduces ageing pigments and promotes physical activity, consistent with increased health.

Impairing bcat-1 expression increases BCAAs. We next questioned whether blocking bcat-1 expression would affect accumulation of its substrates, namely L-leucine, L-isoleucine and L-valine. Consistent with the biochemical role of the enzyme BCAT-1, all three BCAAs were found to be increased by $158 \%$ or more (L-val $+167 \%$, L-ile $+158 \%$, L-leu $+225 \%)$, while L-alanine, L-glutamine and L-glutamate were found to be slightly decreased (Fig. 7i), possibly resembling anaplerotic refuelling of the Krebs cycle, while other amino acids remained unchanged (Supplementary Fig. 2b). The increase in BCAAs was independently confirmed using mass spectrometry-based metabolomics (Fig. 7j). The latter methodology in addition indicated a metabolic shift in bcat-1-impaired worms (Supplementary Data 4), further corroborated by RNA-seq pathway analysis of such nematodes (upregulated: neuro-active ligand-receptor interaction (cel04080); downregulated: TGF $\beta$ signalling pathway (cel04350), oxidative phosphorylation (cel00190), BCAA degradation (cel00280)), both in comparison with wild-type controls, at an age of 5 days.

Increasing BCAA levels promotes $C$. elegans longevity. Based on the findings on increased BCAA levels (Fig. 7i,j), we then exposed wild-type $\mathrm{N} 2$ nematodes to the BCAA L-leucine at a concentration of $5 \mathrm{mM}$, while L-alanine served as a control. While L-alanine had no effect on $C$. elegans lifespan, L-leucine did promote longevity (Fig. 8a, Supplementary Table 4, applies to all subsequent lifespan analyses) however to a lesser extent than bcat-1 RNAi (Fig. 7b). Similar results were obtained for the remaining BCAAs, namely L-isoleucine (Supplementary Fig. 2c) and L-valine (Supplementary Fig. 2d). These findings suggest that impaired bcat-1 expression may promote lifespan, at least in part, by increasing organismal levels of different BCAAs.

We next tested whether and to which extent known transcriptional executers of lifespan-extending interventions may contribute to the phenotype by performing epistasis experiments. While the

Table 1 | Effects of individual RNAis on C. elegans lifespan.

\section{Effect on lifespan}

Shortened

Unchanged

Extended $<5 \%$

Extended $\geq 5 \%$

\section{Upregulated genes}

sma-1, mod-5, mfb-1

mrp-2, cft-1, M116.2, cht-1

Y71G12B.31, ZC373.4

fkh-7, csp-2, lgg-1, Y50C1A.1
Downregulated genes

calu-1, spds-1, ssq-1, act-4, fat-7, act-1

cpn-2, tba-9, ifa-1, ost-1, ssq-4, ssq-3, ifc-1

plk-3, tba-6, ifb-2, F13A7.1, ifb-1, ifc-2, cyb-2.1

bcat-1, T25B9.1, ndk-1, ifd-1, tba-4, ifa-3, try-1, ifp-1 

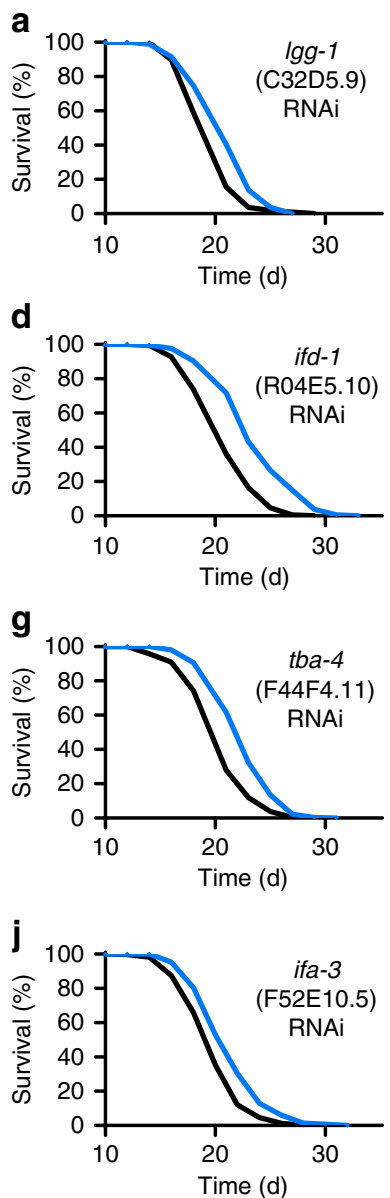

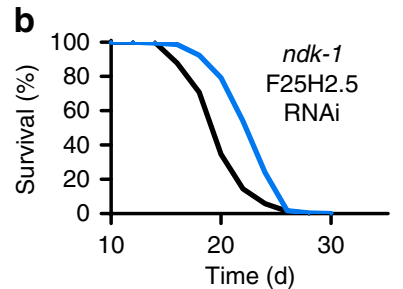

e

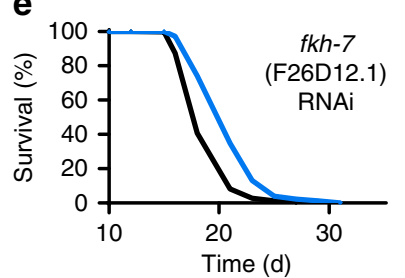

h
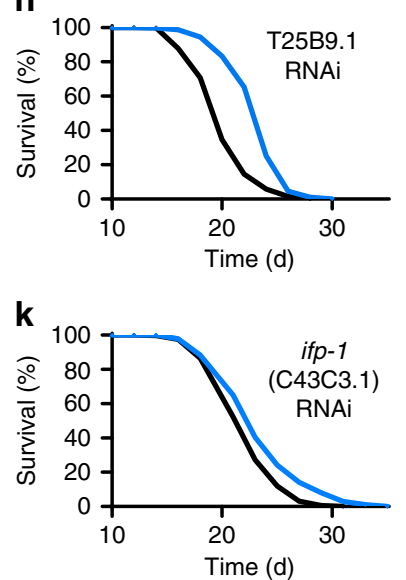

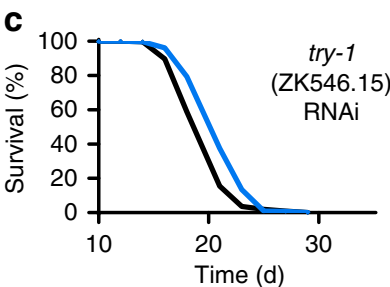

f

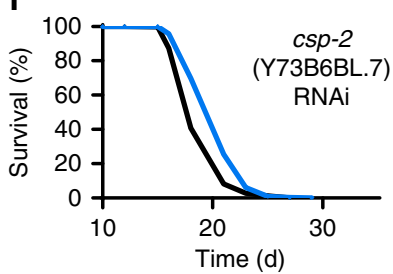

i

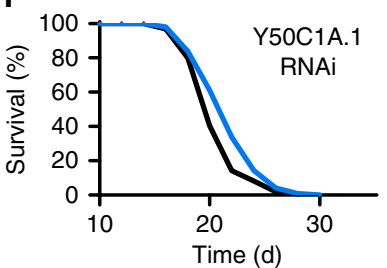

Figure 3 | Lifespan analyses in $\mathbf{C}$. elegans for validation of impact on ageing with significantly increased lifespan ( $\geq \mathbf{5 \%}$ ). (a-k) depict lifespan assays

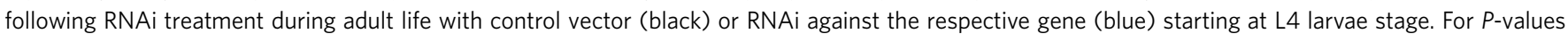
and number of experiments see Supplementary Table 2. Note that results for bcat-1 have been omitted since shown subsequently.

NRF2/SKN-1 pathway appears dispensable for the bcat-1 RNAi- as well as the L-leucine-mediated lifespan extension (Supplementary Fig. 2e,f), both FoxO/Dauer formation 16 (DAF-16) and heatshock factor 1 (HSF-1) appear to be epistatically relevant, since impairment of $h s f-1$ abolished lifespan extension (Fig. 8b, Supplementary Fig. $2 \mathrm{~g}$ ), and impaired daf-16 expression reduced it almost completely (Fig. 8c and Supplementary Fig. 2h). Moreover, application of bcat-1 RNAi to nematodes increases phosphorylation of the $C$. elegans equivalent of the murine Ser326 residue of HSF-1 (Supplementary Fig. 2i-1).

Bcat-1-mediated longevity depends on mTOR activation. Quantifying individual amino acid concentrations following bcat-1 impairment (Fig. 7i) also indicated a global increase $(+38.4 \%)$ in organismal free amino acids. Increased levels of amino acids activate the mTOR pathway, or its $C$. elegans ortholog let-363, respectively. Inhibition of this pathway is known to extend lifespan in different species ${ }^{17}$, being in apparent conflict with our current findings (Figs $7 \mathrm{~b}$ and $8 \mathrm{a}$, Supplementary Fig. 2c,d). We therefore repeated the experiments depicted in Fig. $7 \mathrm{~b}$ in the presence of the mTOR-inhibitor rapamycin and the respective solvent control. As previously observed ${ }^{27}$, rapamycin extended lifespan of C. elegans (Fig. 8d). Interestingly, the effect of bcat-1 on lifespan was reduced by chemically inhibiting LET-363 to the same extent as lifespan was increased by rapamycin alone (Fig. 8d). This unexpectedly indicates that bcat-1 disruption acts lifespan-extending in a let-363-dependent manner (Fig. 8d) paralleled by increased BCAAs and global amino acid levels (Fig. 7i,j).

Neuronal mTOR signalling transduces bcat-1 impairment. Treatment with RNAi is known to affect all tissues of the wild-type Bristol N2 strain except for neurons, while small molecule-based compounds like rapamycin also act on this latter cell type. This together with the findings summarized above raised the hypothesis that non-neuronal bcat-1 interference would generate a cell-nonautonomous small-molecule signal that exerts its lifespan-extending effects in the neuronal compartment in a let-363-dependent manner. To test this, we used the C. elegans TU3311 strain known to respond to RNAi feeding by preferentially impairing expression of the corresponding gene in neurons only ${ }^{28}$. Impairing expression of let-363 in neurons only had no effect on C. elegans lifespan (Fig. 8e), while the effect of reducing bcat-1 in neurons had a strongly reduced, albeit still detectable effect (Fig. 8e). Interestingly, impairing expression of both bcat-1 and let-363 in neurons in parallel almost completely abolished the effect of bcat-1 RNAi on lifespan (Fig. 8e). Given the fact that mTOR transduces availability of anabolic substrates and specifically the BCAA L-leucine in the murine hypothalamus ${ }^{16,29}$, we next analysed a possible involvement of the nematodal ASI neurons which are considered the hypothalamus equivalent in $C$. elegans. To test this, we laser-ablated these two neurons in nematodes and found the effect of bcat-1 RNAi (Fig. 8f) and L-leucine feeding (Fig. 8g) on lifespan to be abolished, while sham-treated worms still responded to the respective intervention (Supplementary Figs 3a, b). Altogether this 

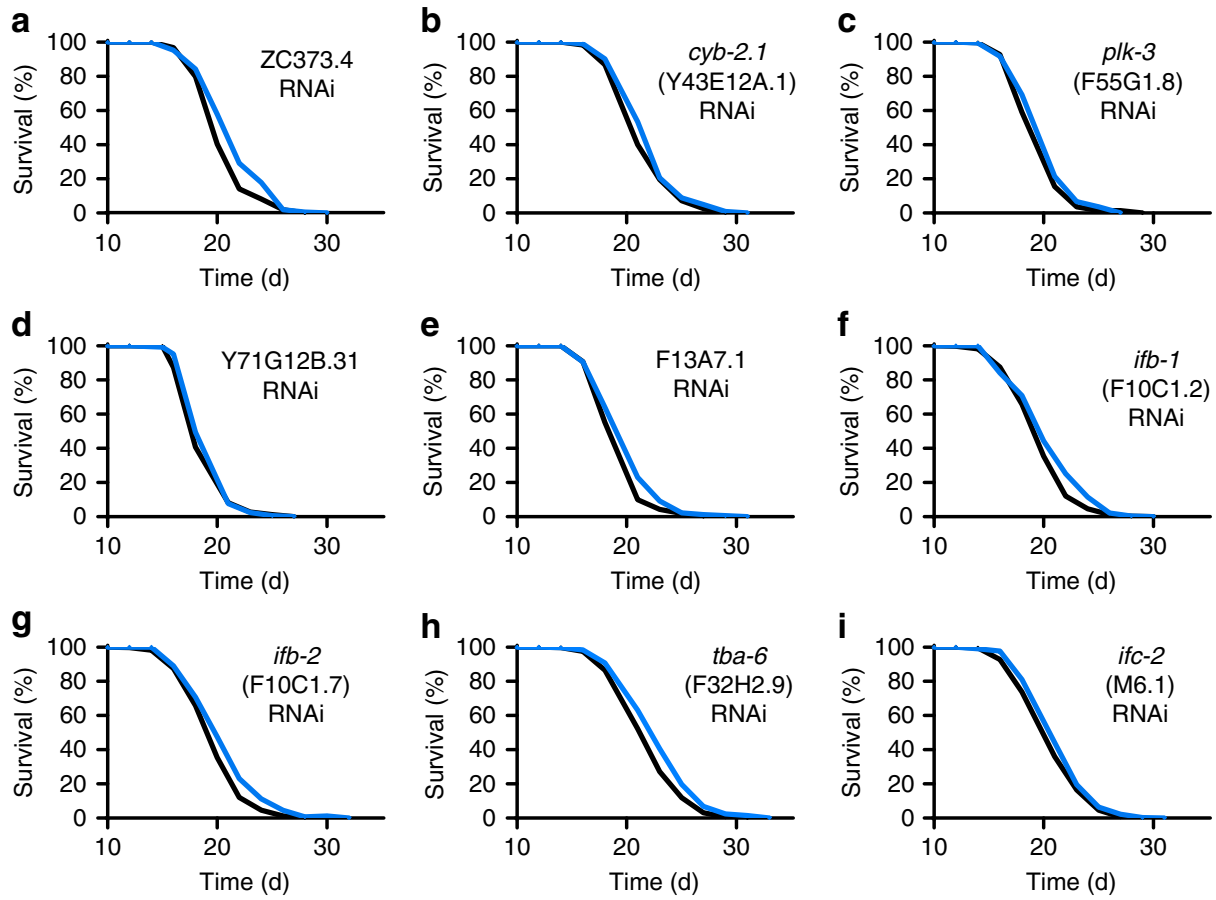

Figure 4 | Lifespan analyses in C. elegans for validation of impact on ageing with significantly increased lifespan ( $<\mathbf{5 \% )}$. (a-i) depict lifespan assays following RNAi treatment during adult life with control vector (black) or RNAi against the respective gene (blue) starting at L4 larvae stage. For P-values and number of experiments see Supplementary Table 2.

indicates that peripheral BCAAs activate neuronal let-363 as well as ASI neuron-specific pathways to exert a lifespan-extending response.

Hypothalamic TGF $\beta$ signalling executes bcat-1 impairment. Given this potentially ASI-specific response (Fig. 8d,e) as well as the RNA-seq-based pathway analysis (Fig. 7l), we next studied the nematodal paralog of mammalian TGF $\beta$, daf-7, as a putative candidate to explain the BCAA-dependent activation of let-363 in neurons, also since daf-7 expression is restricted to ASI neurons ${ }^{30}$ and to the daf-16 pathway ${ }^{31,32}$, while hypothalamic TGF $\beta$ is linked to ageing in mammals ${ }^{33}$. When exposing a strain with a constitutive inactivation of daf-7, namely daf-7 (m62), to bcat-1 RNAi no effect of this treatment was observed (Fig. 8h) opposing the effect in wild-type nematodes (Fig. 7b). This indicates that ASI-specific release of DAF-7 may execute the effect of bcat-1 impairment on lifespan. To further support this, we epistatically tested whether the absence of known DAF-7-receptor genes, namely daf-1 and daf-4, would affect bcat-1 RNAi-mediated lifespan extension. Indeed, the impairment of either daf-1 (Fig. 8i) or daf-4 (Fig. 8j) expression completely inhibited the bcat-1 RNAi effects on lifespan: while daf-1-mutated worms were long-lived, and so were nematodes treated with daf-4 RNAi, addition of bcat-1 RNAi would not extend lifespan further, indicating that bcat-1 and daf-1/daf-4 epistatically share a downstream pathway. Notably and since daf-4 RNAi does not affect neurons, while daf7 expression is limited to ASI neurons ${ }^{30}$, this also indicates that this TGF $\beta /$ daf-7 signal qualifies as a cell-non-autonomous feedback loop linking peripheral signals that activate neuronal (and potentially ASI-specific) mTOR back to the periphery.

Overexpression of bcat-1 impairs lifespan and fecundity. We next questioned whether overexpression of bcat-1 would have opposing effects on lifespan, particularly in comparison to states of impaired expression, as shown above (Fig. 7b). To this purpose, we expressed a bcat-1-complementary DNA (cDNA) $3^{\prime}$-/C-terminally fused to a green fluorescent protein (GFP)cDNA under the control of the endogenous bcat-1 promoter. Fluorescent microphotographs indicated a reduction of BCAT-1 expression with increasing age (Fig. 9a), consistent with the gene expression levels of the endogenous bcat-1 in wild-type worms (Fig. 7c). Importantly and opposing the findings on bcat-1 RNAi (Fig. 7b), overexpression of bcat-1 shortens C. elegans lifespan (Fig. 9b), further supporting a regulatory role in the regulation of lifespan. Moreover, overexpression of bcat-1 decreased fertility of nematodes (Fig. 9c) that, from an evolutionary perspective, suggests a selective advantage of low bcat-1 expression levels, notably independent of ageing.

HLH-15 is a transcriptional regulator of bcat-1. Based on the fact that reduced versus increased levels of BCAT-1 exhibit opposing effects on lifespan (Figs $7 b$ versus 9a), we next questioned whether and how bcat-1 expression may be systemically controlled during physiological ageing. We in silico analysed a 1,000 base-pair promoter fragment upstream of the bcat-1 start codon, and identified a transcription factor named helix-loop-helix factor 15 ( $h l h-15)$ to (i) show the highest $(n=3)$ number of binding sites within this promoter fragment and to have (ii) the highest $P$-value for binding probability (Markov Chain model, $P=8.7 \mathrm{e}-07$; Supplementary Table 5).

To confirm the in silico prediction that HLH-15 may control the expression of bcat-1, we applied RNAi against hlh-15 to wild-type nematodes and found expression of bcat-1 to be reduced by $>50 \%$ (Fig. $9 \mathrm{~d}$ ). When analysing the RNA-seq data obtained from C. elegans during physiological ageing (Fig. 2), we found expression levels of bcat-1 (Fig. 9e, Pearson $r=-0.9964$ ) and hlh-15 (Fig. 9e, Pearson $r=-0.9693$ ) to be correlated by trend (Pearson $P=0.0537$ ), suggesting that HLH-15 may regulate bcat-1 expression during physiological ageing. To lastly test this, we have performed the corresponding epistasis experiments: 

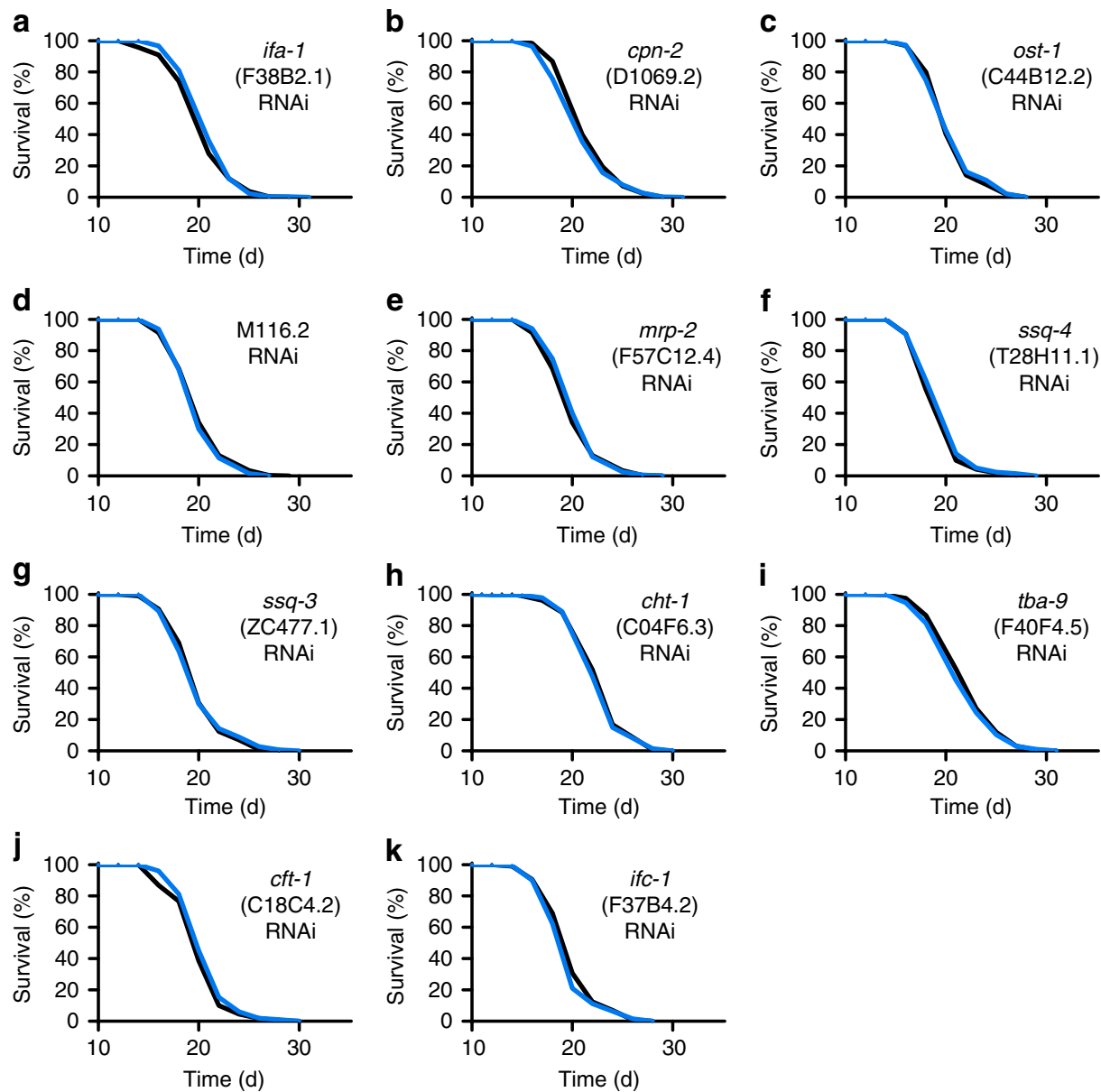

Figure 5 | Lifespan analyses in C. elegans for validation of impact on ageing with no significant effect on lifespan. (a-k) depict lifespan assays following RNAi treatment during adult life with control vector (black) or RNAi against the respective gene (blue) starting at L4 larvae stage. For P-values and number of experiments see Supplementary Table 2.

RNAi against bcat-1 or against hlh-15, respectively, extended nematodal lifespan to the exact same extent (Fig. 9f). Co-application of both RNAis did show the same effect as each RNAi individually (Fig. 9f), altogether suggesting that HLH-15 and BCAT-1 may actively synergize in the regulation of physiological ageing (Fig. 9g).

\section{Discussion}

Increased BCAA catabolism and specifically increased activity of the corresponding enzyme, BCAT, has been linked to various pathological states, including accelerated growth of malignant gliomas ${ }^{34}$, decreased sepsis survival ${ }^{35}$ and increased accumulation of liver $\mathrm{fat}^{36}$, the latter being linked to a number of metabolic diseases ${ }^{37}$. Consistently, systemic disruption of one $B C A T$ isoform ${ }^{38}$, namely $B C A T m$, in mice increases energy expenditure and reduces body weight ${ }^{39}$.

More specifically, L-leucine is capable of reducing food uptake in rodents when injected into the hypothalamus, notably depending on neuronal mTOR (refs 16,29). By contrast, others have linked hypothalamic BCAAs to increased insulin resistance, providing a potential link to type 2 diabetes ${ }^{40,41}$. Since nematodal ASI neurons are considered the homologue of the mammalian hypothalamus $^{42}$, and ablation of these two neurons abolishes the effect of both L-leucine (Fig. 8g) and bcat-1 RNAi (Fig. 8f), the effect of increasing organismal L-leucine levels on lifespan (Fig. 7b,i,j) may be translatable into mammals. Indeed, feeding increased amounts of BCAAs to middle-aged mice did improve healthspan depending on induction of endothelial nitric oxide synthase $^{43}$, an enzyme not known in C. elegans ${ }^{44}$. Moreover, other hypothalamic signals have been recently linked to the regulation of lifespan in rodents ${ }^{45,46}$, while mTOR signalling has not been tested in this regard.

Global inhibition of mTOR is generally considered to promote lifespan in different species ${ }^{17}$ including C. elegans $^{27}$ (also confirmed by our control findings, Fig. 8d). By contrast and unexpectedly, we here find that activation of mTOR in nematodal neurons due to a peripheral BCAA signal promotes lifespan (Fig. 7e) and parameters of healthspan (Fig. 7f,h) without affecting fecundity (Fig. $7 \mathrm{~g}$ ). Exerting the BCAA signal in neurons only still exerts an effect on lifespan, however to a reduced extent (Fig. 8e). Activation of mTOR is known to induce HSF-1 (ref. 47), and the latter suppresses daf-7 (ref. 48), consistent with our findings which link mTOR activation to suppression of $d a f-7 / T G F \beta$ signalling (Figs $7 \mathrm{l}$ and $8 \mathrm{~h}-\mathrm{j}$ ). Since $d a f-7$ is expressed in ASI neurons only ${ }^{30}$, while its receptors daf- 1 and daf- 4 act in the periphery ${ }^{49}$, daf-7/ TGF $\beta$ may now be considered to act cell-non-autonomous as a neuro-endocrine hormone that impairs lifespan in otherwise healthy animals in response to evolutionary conserved pathways and amino acid signals derived thereof (Fig. 9g).

\section{Methods}

Chemicals. All chemicals were obtained from Sigma-Aldrich (Munich, Germany) unless stated otherwise. 

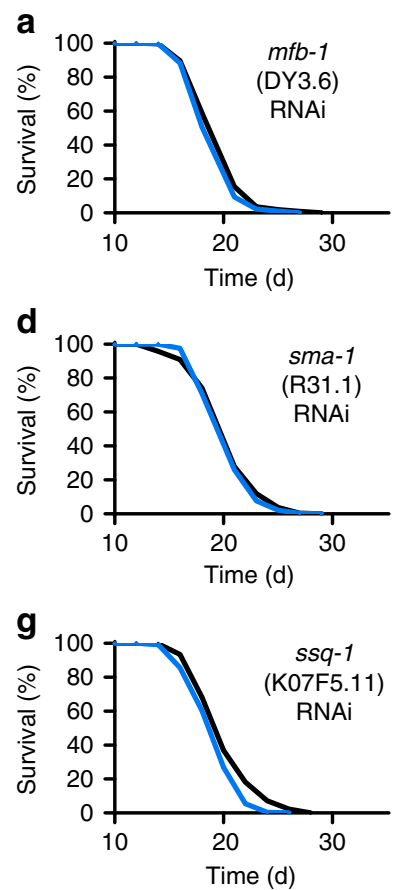

b
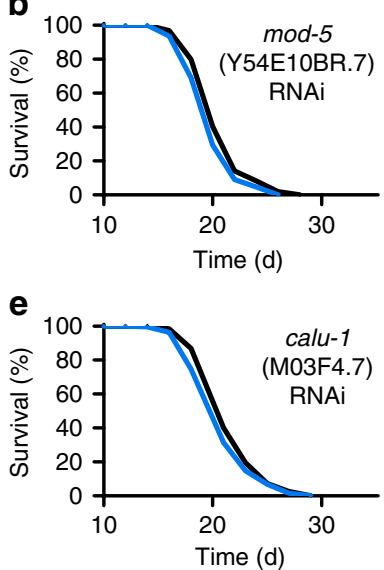

h

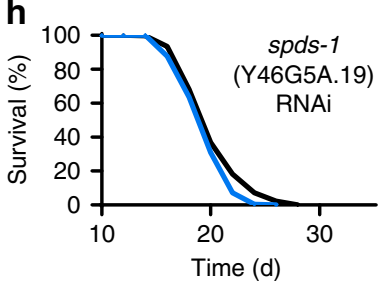

C

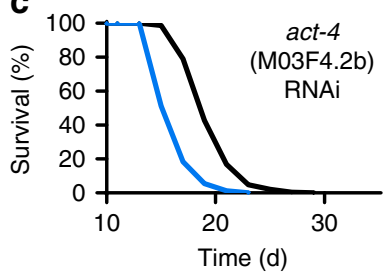

f

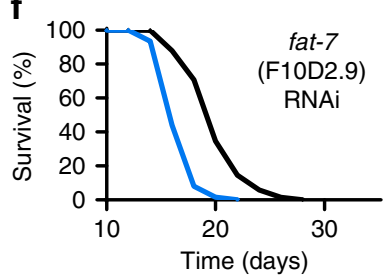

i

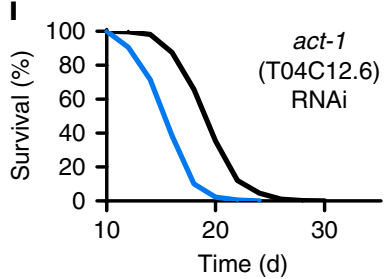

Figure 6 | Lifespan analyses in C. elegans for validation of impact on ageing with significantly shortened lifespan. (a-i) depict lifespan assays following RNAi treatment during adult life with control vector (black) or RNAi against the respective gene (blue) starting at L4 larvae stage. For P-values and number of experiments see Supplementary Table 2.

C. elegans strains and maintenance. C. elegans strains used for this publication were provided by the Caenorhabditis Genetics Center (University of Minnesota, USA). Nematodes were grown and maintained on Nematode growth media (NGM) agar plates at $20^{\circ} \mathrm{C}$ using E. coli OP50 bacteria as food source ${ }^{50}$. After plates were poured and dried, they were sealed and stored at $4^{\circ} \mathrm{C}$. Freshly prepared E. coli were spotted on plates on the previous evening and allowed to dry and settle overnight. For all experiments we used Bristol N2 wild-type except the following we received from CGC: EU31 skn-1(zu135), PS3551 hsf-1(sy441), CF 1038 daf-16(mu86), CX3596 kyIs128[str-3::GFP]; lin-15B(n765), TU3311 uIs60[unc119p::YFP + unc-119p::sid-1], SJ4100 zcIs13[hsp-6::GFP], DR62 daf-7(m62)and DR40 daf-1 $(m 40)$.

Sample preparation for physiological worm ageing experiments. Freshly prepared bacteria OP50 were spotted on $10 \mathrm{~cm}$ NGM agar plates on the previous evening and allowed to dry and settle overnight. Synchronized, young adult worms (64 $\mathrm{h}$ after synchronization) were transferred to fresh plates using S-Buffer ${ }^{51}$. For maintaining synchronized populations in long-term experiments, worms were daily washed off the plates to $15 \mathrm{ml}$ tubes, allowed to settle and washed until the supernatant was free of progeny. The clean worm pellet was transferred to freshly prepared treatment plates. Worms were pelleted and frozen at an adult age of 1,10 and 20 days.

RNAi-mediated gene knockdown and compound treatment. For RNAi gene knockdown experiments we applied E. coli HT115 to the worms as previously described $^{52}$. The clones for act-1,daf-4, cyb-2.1, ssq-1, F13A7.1, ssq-4, T25B9.1, ifa-1, ifa-3, ifb-2, ifc-2, ifd-1, ndk-1, plk-3, try-1, calu-1, fat-7, spds-1, cpn-2, tba-4, tba-6, mfb-1, lgg-1 and hlh-15 RNAi were obtained from feeding RNAi ORF library v1.1 (Thermo Fisher Scientififc, Waltham, MA, USA). The clone for bcat-1, let-363, cht-1, ost-1, ifb-1, ifp-1, sma-1, tba-9, mod-5, Y50C1A.1 and ZC373.4 derived from Ahringer library (Source BioScience, Nottingham, UK). The remaining clones were generated from PCR products of genomic DNA and were cloned into control vector L4440 using its EcoRV restriction site. Primer sequences are listed in Supplementary Table 6. All (that is, also commercially obtained) clones were sequenced prior use.

The bacteria were spotted on NGM plates containing additionally $1 \mathrm{mM}$ isopropyl- $\beta$-D-thiogalactoside, $100 \mu \mathrm{g} \mathrm{ml}^{-1}$ ampicillin and, if required, $12.5 \mu \mathrm{g} \mathrm{ml}^{-1}$ tetracycline (all from Applichem, Darmstadt, Germany). After plates were poured and dried, they were sealed and stored at $4{ }^{\circ} \mathrm{C}$. Freshly prepared bacteria were spotted on plates on the previous evening and allowed to dry and settle overnight. Incubations with compounds started $64 \mathrm{~h}$ after synchronization of the population, by washing the synchronized, young adult worms and then transferring them to the respective treatment plates using S-Buffer ${ }^{51}$. For maintaining synchronized populations in long-term experiments, worms were daily washed off the plates to $15 \mathrm{ml}$ tubes, allowed to settle and washed until the supernatant was free of progeny. The clean worm pellet was transferred to freshly prepared treatment plates.

For compound treatments, all agar plates were prepared from the same batch of NGM agar, whereas treatment plates were supplemented with L-leucine, L-alanine (both $5 \mathrm{mM}$ final concentration) or water as solvent control. Rapamycin was used in a concentration of $100 \mu \mathrm{M}$ and dimethylsulphoxide (DMSO) served as solvent control. For all experiments with amino acid supplementation only heat-inactivated bacteria were used to avoid influences on bacterial metabolism. The bacteria were heat-inactivated for $45 \mathrm{~min}$ at $65^{\circ} \mathrm{C}$ in a shaking incubator and resuspended and concentrated $(20 \times)$ in S-buffer containing $5 \mu \mathrm{g} \mathrm{ml}^{-1}$ and $10 \mathrm{mM} \mathrm{MgSO}_{4}$. The experimental procedures are the same as described above for RNAi treatment experiments.

Nematodal lifespan assays. All lifespan assays were performed at $20^{\circ} \mathrm{C}$ according to standard protocols and as previously described ${ }^{52}$. Briefly, a C. elegans population was synchronized as described above at day 0 of the lifespan. $64 \mathrm{~h}$ after egg preparation around 100 nematodes were manually transferred to fresh incubation plates containing the respective compounds. Experiments were conducted in triplicates. For the first 10 days, worms were transferred every day and afterwards every second day. Nematodes that show no reaction to gently stimulation were scored as death. Those animals that crawled off the plates or display non-natural death due to internal hatching were censored.

D. rerio. Zebrafish of the TüAB strain were kept in groups of 20-30 animals under standard husbandry conditions. Skin tissue from male zebrafish at the age of 5 months $(n=12), 24$ months $(n=12)$ and 42 months $(n=6)$ was dissected and stored in RNAlater (Qiagen, Hilden, Germany) at $-80^{\circ} \mathrm{C}$. Total RNA was isolated with TRIzol (Life Technologies, Darmstadt, Germany) according to the instructions of the manufacturer.

M. musculus. Young (2 months), mature (15 months) and aged (30 months) old mice were deeply anaesthetized with isoflurane anaesthesia $(2.5 \%$ in a mixture of 3:1 $\mathrm{N}_{2} \mathrm{O}: \mathrm{O}_{2}$ ). One square $\mathrm{cm}$ hairless abdominal skin was taken and snap frozen. The isolated skin was homogenized in $500 \mu$ l QIAzol (Qiagen) by subsequently adding $100 \mu \mathrm{l}$ chloroform. Following phase separation, the aqueous phase was transferred into a fresh tube, then 0.16 volume $\mathrm{NaAc}(2 \mathrm{M}, \mathrm{pH} 4.0)$ and 1.1 volume isopropanol were added. The RNA was precipitated by centrifugation and the pellet was washed with $75 \%$ ethanol. Total RNA was resuspended in $20 \mu \mathrm{l}$ water and stored at $-80^{\circ} \mathrm{C}$ until use. 


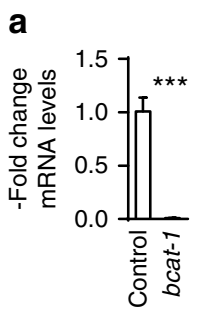

b

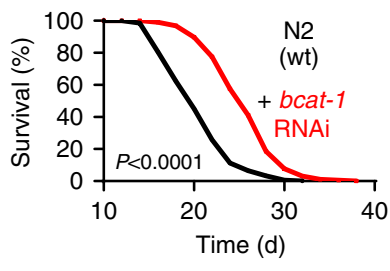

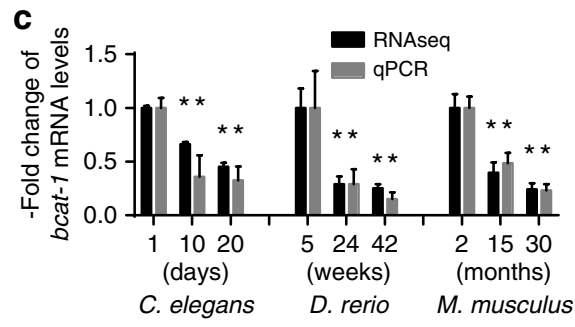

d

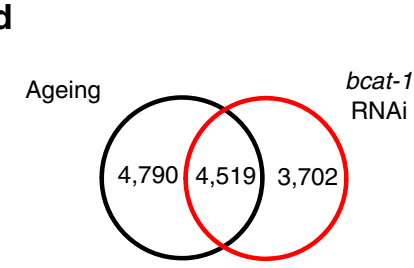

e
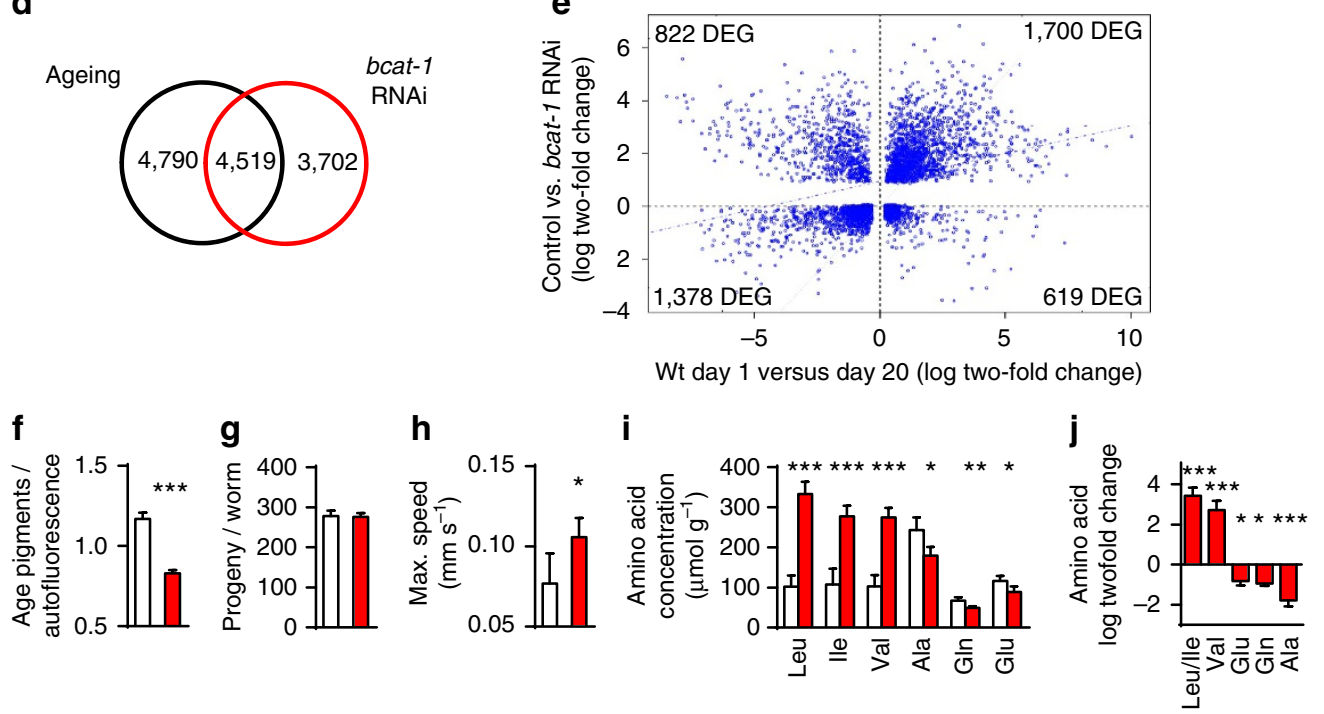

Figure 7 | Validation and characterization of bcat-1 as an ageing-related gene. (a) Shows bcat-1 RNA levels in whole-worm RNA extracts after treatment of $C$. elegans with RNAi against bcat-1 (red bar) versus control RNAi (open bar; $P<0.001$, Student's $t$-test, $n=3$ ). (b) Depicts the effect of bcat-1 RNAi (red) versus control (black) on lifespan $(P<0.0001$, log-rank test, $n=3$ ). (c) Depicts qPCR results (grey) in comparison with RNA-seq results (black; samples as depicted in Fig. 1c; $P<0.05$ versus first time point; one-way ANOVA, $n=3$ ). (d) shows a Venn analysis of transcripts that are regulated by physiological ageing (blue) and bcat-1 RNAi treatment (red), respectively. (e) Shows transcript levels as in $\mathbf{d}$ quantitatively $(P<0.001$, correlation, $n=3)$. (f-I) Depict bcat-1 RNAi-treated (red) versus control (open) nematodes regarding (f) ageing pigments $(P<0.001$, Student's $t$-test, $n=8),(\mathbf{g})$ progeny $(P=0.7$, Student's $t$-test, $n=10)$, (h) maximum crawling speed $(P<0.05$, Student's $t$-test, $n=5)$, as well as changes in whole-worm amino acid concentrations as determined by (i) HPLC ( ${ }^{\star} P<0.05,{ }^{\star \star} P<0.01,{ }^{\star \star \star} P<0.001$ versus control, one-way ANOVA, $\left.n=4\right)$, and $(\mathbf{j})$ mass spectrometry ( $P<0.05$, ${ }^{\star \star} P<0.01$, ${ }^{\star * \star} P<0.001$ versus control, one-way ANOVA, $\left.n=4\right)$. Error bars represent the means \pm s.d.

Breeding and housing conditions. The study was carried out on male C57BL/6J mice (Jackson Laboratories). Animals of given ages were raised in our own facilities. All animal procedures were approved by the local government (Thueringer Landesamt, Bad Langensalza, Germany) and conformed to international guidelines on the ethical use of animals. All mice were maintained in a specific pathogen-free environment at room temperature $\left(22^{\circ} \mathrm{C}\right)$ at $68 \%$ humidity and light/dark $(12 \mathrm{~h} / 12 \mathrm{~h})$ cycles with access to water and food (V1534-300, SsniffSpezialdiäten $\mathrm{GmbH}$, Soest, Germany), ad libitum and were tested negative for parasites and other routine pathogens.

RNA-seq. RNA integrity was determined by Agilent's Bioanalyzer 2100 (with RNA 7500 kit, both Agilent Technologies, Santa Clara, CA, USA).

M. musculus and C. elegans physiological ageing samples. Total RNA $(2.5 \mu \mathrm{g})$ was used with Illumina'sTruSeq RNA sample prep kit v2 following the manufacturer's instruction. Illumina $50 \mathrm{bp}$ single reads (SR) were obtained using the HiSeq2000 by multiplexing four samples per lane. Sequencing resulted in around 40-50 mio reads per sample. The sequence information was extracted using Illumina's supported CASAVA v1.7 as FastQ format.

D. rerio. Total RNA ( $5 \mu \mathrm{g})$ was used for preparation of multiplex libraries using Illumina's mRNA-Seq sample prep kit (Illumina, San Diego, CA, USA) following the manufacturer's instruction. Libraries were sequenced in one per lane using Illumina's Genome Analyzer (GAIIx) in SR mode creating reads with a length of $76 \mathrm{bp}$. Sequencing resulted in around 30 mio reads per samples. Sequence information was extracted in FastQ format using the Illumina's supported GAPipeline v1.5.

C. elegans bcat-1 RNAi perturbation samples. Total RNA $(1 \mu \mathrm{g})$ was used for library preparation using TruSeq RNA sample prep kit v2 following the manufacturer's instruction. Sequencing was done on a HiSeq2500 in SR/50 bp/high output mode. Libraries were multiplexed in five per lane. Sequencing resulted in around 35-45 mio reads per sample. The sequence information was extracted using Illumina's supported bcl2FastQ v1.8.4 as FastQ format.
RNA-seq data analysis. Normal ageing samples. The resulting FastQ files were mapped using Bowti ${ }^{53}$ versus the respective genomic sequences and a splice site data set created using UCSC's RefSeq annotation for each species. Counting of uniquely mapped reads and assignment to RefSeq transcripts/genes was performed using R Statistical Language and Bioconductor. Afterwards, reads per kilobase transcript per million reads (RPKM) values ${ }^{54}$ were calculated for each transcript and gene from the corresponding RefSeq annotation. For each species DEG were identified using the DESeq ${ }^{55}$, edgeR ${ }^{56}$, and the baySeq ${ }^{57}$ packages. Those genes showing statistically significant differences (FDR adjusted $P<0.05$ ) by DESeq and edgeR at least between two time points or by the baySeq test over the three time points were regarded as DEGs (C. elegans: $n=3,608 ; D$. rerio: $n=1,721$; and $M$. musculus: $n=339$ ). Next, orthology relations between genes of the three species were obtained using EnsemblCompara and the orthology R package ${ }^{58}$ to facilitate the cross-species comparison. Since the individual species exhibit different lifespan, the gene expression time courses were rescaled, followed by a combined fuzzy c-means clustering with the exponent $m=2$ of the orthologous DEG expression profiles ${ }^{59}$, to identify common time courses. The optimal number of six clusters was estimated by the vote of several cluster validity indices, which capture different aspects of a clustering structure ${ }^{60}$. By intersecting the resulting clustered gene sets with the orthology relations extracted previously, gene sets with similar temporal pattern across all three species were identified.

For the analysis of differentially regulated metabolic pathways in the normal ageing samples, C. elegans genes were mapped to metabolic pathways using the PathwayTools software ${ }^{61}$. A reaction was assumed differentially regulated if at least one enzyme catalysing this reaction was significantly differentially expressed between young and old worms. Pathways were assumed significantly differentially regulated if a hypergeometric test of differentially regulated reactions yielded a FDR adjusted $P$-value $<0.05$

C. elegans bcat-1 RNAi perturbation samples. FastQ files were mapped using Tophat (v2.0.6) ${ }^{62}$ versus the reference genome WBcel235.74 obtained from Ensembl. Uniquely mapped reads were counted for all genes using 

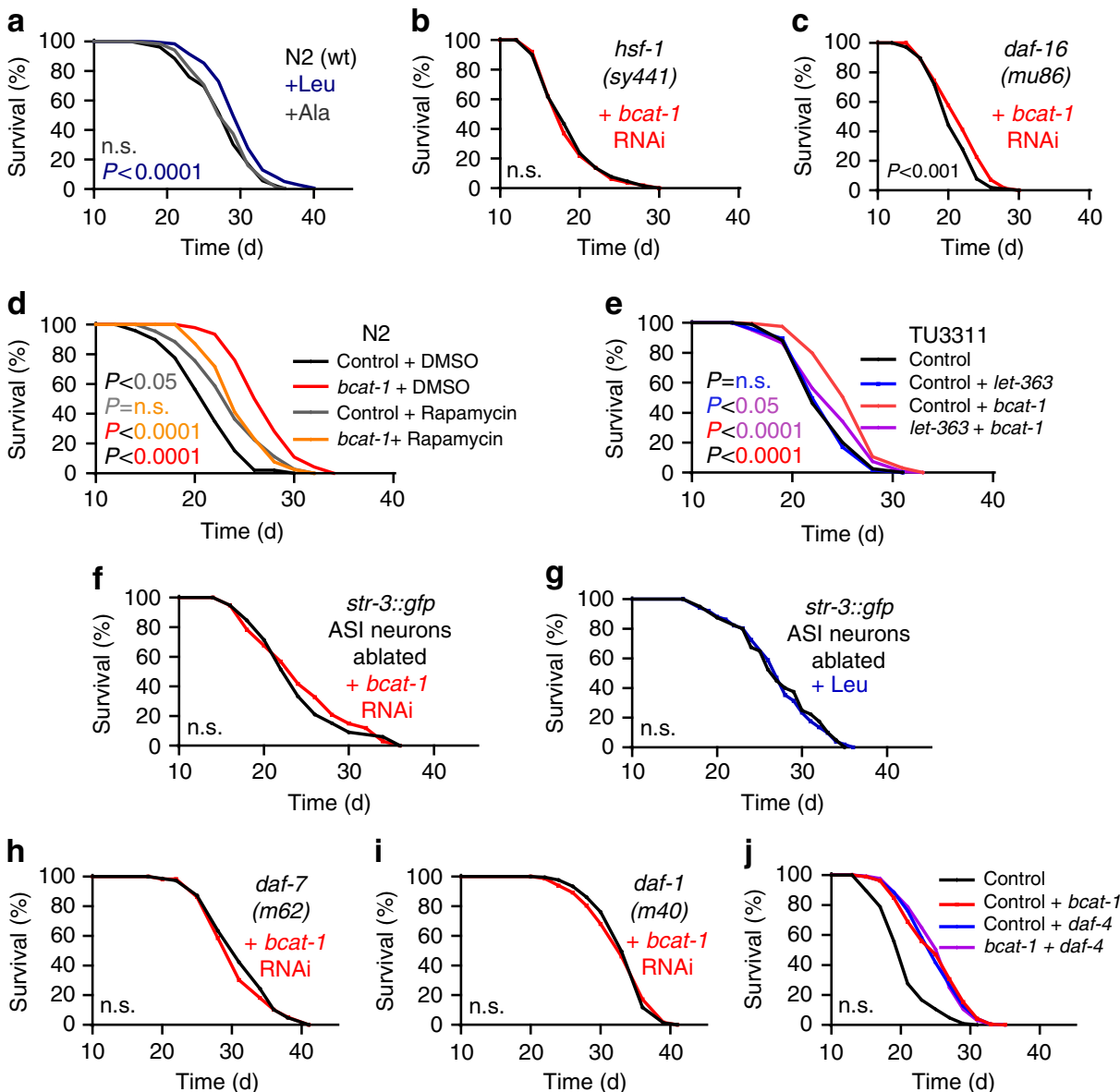

Figure 8 | Increased BCAAs act through neuronal LET-363/mTOR and peripheral DAF-7/TGF $\beta$ signaling to extend lifespan. (a) Shows

lifespan data of wild-type nematodes without treatment (black), as well as, exposure to L-alanine (grey, $5 \mathrm{mM}$ ) and L-leucine (blue, $5 \mathrm{mM}$ ) for their entire adult lifespan, maintained on non-metabolizing bacteria $(P=0.24$ for $\mathrm{L}$-ala and $P<0.0001$ for L-leu both versus control, log-rank test, $n=3$ ). (b,c) Show effects of bcat-1 RNAi (red) on lifespan in strains mutant for (b) hsf-1 $(P=0.67$, log-rank test, $n=3)$ and $(\mathbf{c})$ daf-16 $(P<0.001$, log-rank test, $n=3)$. (d) Depicts the effects of the mTOR-inhibitor rapamycin $(100 \mu \mathrm{M})$ on wild-type worms (grey versus black) versus the lack of effect of non-neuronal bcat- 1 RNAi in the presence of rapamycin $(100 \mu \mathrm{M})$, all on lifespan $(P<0.05$ for control RNAi/rapamycin versus control RNAi/DMSO, $P=0.07$ for bcat-1 RNAi/ rapamycin versus control RNAi/rapamycin, $P<0.0001$ for bcat-1 RNAi/rapamycin versus bcat-1 RNAi/DMSO, $P<0.0001$ for bcat-1 RNAi/DMSO versus control RNAi/DMSO, log-rank test, $n=3$ ). (e) Depicts the effects of neuronal bcat-1 RNAi on lifespan in the presence (purple) and absence (red) of neuronal RNAi against let-363/mTOR ( $P=45$ for control/let-363 RNAi versus control RNAi, $P<0.05$ for control/let-363 RNAi versus let-363/bcat-1 RNAi, $P<0.0001$ for control/bcat-1 RNAi versus let-363/bcat-1 RNAi, $P<0.0001$ for control RNAi versus control/bcat-1 RNAi, log-rank test, $n=3$ ). (f, $\mathbf{g}$ ) Depict the lack of effect of $(\mathbf{f})$ peripheral bcat-1 RNAi $(P=0.73$, log-rank test, $n=3)$ and $(\mathbf{g})$ L-leucine supplementation $(P=0.96$, log-rank test, $n=3)$ on an ASIablated reporter strain. $(\mathbf{h}-\mathbf{j})$ Show the lack of effect of bcat-1 RNAi on strains mutant for $(\mathbf{h})$ daf-7 $(P=0.15$, log-rank test, $n=3)$ and $(\mathbf{i})$ daf- $1(P=0.84$, log-rank test, $n=3$ ), as well ( $\mathbf{j}$ ) in the co-presence (purple) or absence (red) of RNAi against daf- 4 ( $P=0.8$ for control/bcat- 1 RNAi versus daf- $4 /$ bcat- 1 RNAi, $P<0.0001$ for all treatments versus control, log-rank test, $n=3$ ). For $P$-values and number of repetitions see Supplementary Table 4.

featureCounts ${ }^{63}$. RPKM values were computed using exon lengths provided by featureCounts and the sum of all mapped reads per sample. DEG were identified using the DESeq and edgeR. Generally applicable gene set enrichment for pathway analysis (GAGE) ${ }^{64}$ was used to detect significantly regulated Kyoto Encyclopedia of Genes and Genomes (KEGG) pathways (FDR adjusted $P<0.05$ ).

To evaluate whether the probability for the intersection of two gene expression sets is significantly different from the intersection of random sets we applied a Monte Carlo-based test. We first randomly generated subsets out of our two universe lists of all genes which were measured in both data sets according the number of genes of our real data sets. Then, we counted the number of genes in these random intersections for 1,000 iterations which resulted in a distribution of random intersection values. If the observed intersection value was $>95 \%$ quantile we considered our intersection of genes to be significant.

Reverse transcriptase qPCR (RT-qPCR). Reverse transcription of RNA into cDNA was generated with the iScript cDNA synthesis kit (Bio-Rad, Munich, Germany) and qPCR was carried out with the SybrGreenERqPCRSupermix (Life Technologies) for iCycler. All PCR reactions were performed in triplicates and negative controls were always included. Ct-values of bcat-1 were normalized to two reference genes (for $C$ elegans: $c d c-42$ and pmp-3; for zebrafish: tbp and insra; for mouse: gapdh and $h m b s$; see Supplementary Table 7 for sequences). Determination of age-specific

expression and statistical analysis was carried out using the relative expression software tool $^{65}$.

Bcat-1 RNAi perturbation C. elegans. Adult wild-type worms were treated for $48 \mathrm{~h}$ with control vector L4440 or bcat-1 RNAi, respectively.

Laser ablation of ASI neurons. The laser ablation of ASI neurons was performed as described $^{42,66}$ and as follows: for focused laser ablation the output laser beam of a UV pulsed laser (diode-pumped, Q-Switched Frequency-Tripled Laser System: Triton; TEM00, $349 \mathrm{~nm}$; maximum power $1 \mathrm{~W}$, pulsed; repetition rate: 1-1,000 Hz; pulse width: <15 ns; pulse energy: adjustable from 1 to $200 \mu$ J; Spectra Physics, Darmstadt, Germany) was expanded by a telescope system and was coupled into a confocal laser scanning microscope (LSM510) via epi-fluorescence illumination path. The laser beam was focused into the object plane by a Zeiss Plan-Neofluar 100/1.30 oil objective (spot diameter $<500 \mathrm{~nm}$ ) after reflection by a dielectric mirror (Laser Optik,

Germany). The dielectric mirror is placed on the empty laser scanning position of the fluorescence reflector slider and transmits the scanning lasers as well as the emitted 
a

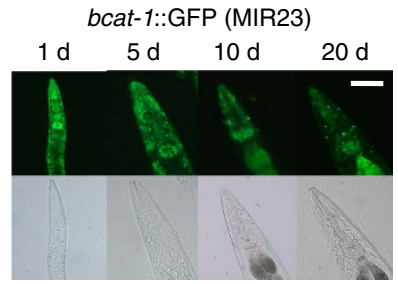

b

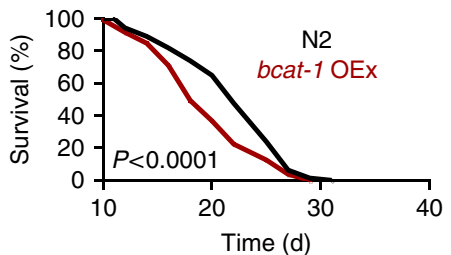

c

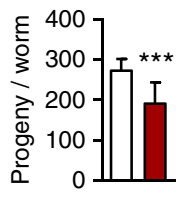

d

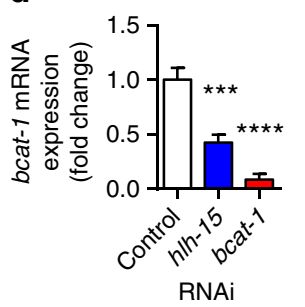

e

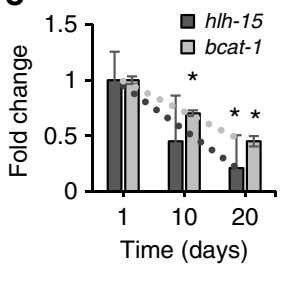

f

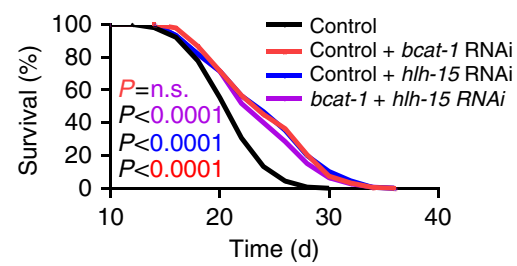

g

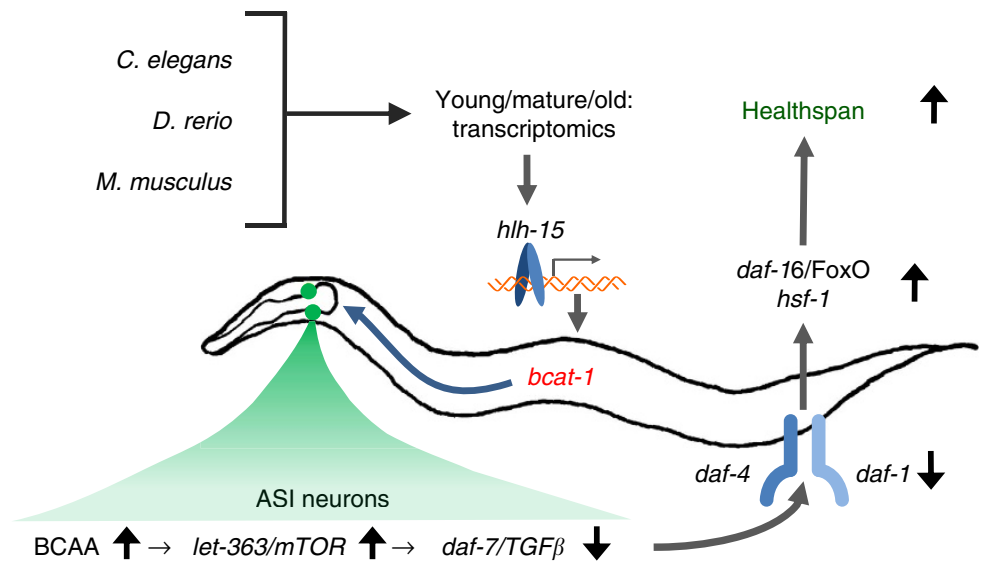

Figure 9 | Transcriptional control of bcat-1-mediated regulation of lifespan. (a) Fluorescent microscopy of nematodes transgenically expressing bcat-1 fused to GFP under the control of the endogenous bcat-1 promoter, at different ages (scale bar, $100 \mu \mathrm{m}$ ). (b) Shows the effect of bcat- 1 overexpression on lifespan $\left(P<0.001\right.$, log-rank test, $n=3$ ). (c) Depicts fertility as reflected by the number of eggs ( ${ }^{\star \star \star} P<0.001$, Students's $t$-test, $\left.n=10\right)$. (d) Depicts transcript levels of bcat- 1 in the presence of control RNAi (white), RNAi against $h / h-15$ (blue) and bcat-1 (red) in wild-type nematodes ( ${ }^{\star \star \star} P<0.001$, ${ }^{\star \star \star \star} P<0.0001$ versus control, one-way ANOVA, $n=4$ ). (e) Shows transcript levels of bcat-1 (black) and hlh-15 (grey) during physiological ageing in wild-type worms ( ${ }^{\star} P<0.05$, oneway ANOVA, Pearson correlation Fisher $Z, P=0.053, n=3$ ). (f) Depicts the effects of control RNAi (black), control RNAi combined with bcat-1 RNAi (red; $P<0.0001$ versus control, log-rank test, $n=3$ ), control RNAi combined with $h / h-15$ RNAi (blue; $P<0.0001$ versus control, log-rank test, $n=3$ ) and bcat-1 RNA combined with $h / h-15$ RNAi (purple, epistasis; $P<0.0001$ versus control, $P=0.08$ versus control/bcat- 1 RNAi, log-rank test, $n=3$ ) on $C$. elegans lifespan. (g) Summarizes the effects of $h / h-15$-controlled bcat-1 expression, or BCAA supplementation, on neuronal let-363/daf-7 signaling looping back to the periphery to control lifespan. For P-values and number of repetitions see Supplementary Table 4. Error bars represent the means \pm s.d.

fluorescence and reflects the pulsed laser beam of Triton. Thus the imaging functions of the laser scanning microscope (LSM) are not reduced. Before entering the microscope, laser pulse energy was reduced by a gradient position dependent attenuator (Laser Optik) to $80 \%$.

Paralyzed post-L4 nematodes that expresses GFP from a ASI-specific str-3 promoter (kyIs128[str-3::gfp]) were irradiated by several laser pulses at laser energy of $4 \mu \mathrm{J}$. The live-cell damage was recorded and controlled by time series function of the Zeiss laser scanning microscope which is equipped with an Argon ion laser and emission filter sets for the detection of EGFP signals (BP530/20) and the Zeiss LSM software version 3.2. Immediately after ablation, worms were transferred to the corresponding treatment plates and analysed for life expectancy according to the described lifespan protocol above.

Protein quantification. Protein content in nematodes and cells was determined by the Bradford method ${ }^{67}$ or the bicinchoninic acid (BCA) method ${ }^{68}$. Assays were performed in 96-well plates using commercial available kits (Bio-Rad Laboratories AG, Cressier, Switzerland, and Thermo Scientific, Waltham, MA, USA). Absorbance was measured in a micro-plate reader (Fluostar Optima, BMG Labtech, Offenburg, Germany).

Determination of amino acid concentrations by HPLC. Frozen worm pellets have been ground with $200 \mu \mathrm{lBS}(\mathrm{pH}=7.4)$ and de-proteinized with sulfo- salicylic acid (final concentration 2\%; Sigma-Aldrich, St Louis, MO, USA). The cell debris and protein precipitate were separated by spinning. For the measurements $30 \mu \mathrm{l}$ of supernatant was used.

Determination of amino acid concentration was carried out on a Biochrome $30+$ Amino Acid Analyzer (Biochrom, Cambridge, UK) following manufacturer's instructions. This standard method in clinical diagnostics is based on separation on a retention column with lithium citrate buffer of different $\mathrm{pH}$. The post-column derivatization with ninhydrin enables photometric detection at 570 and $440 \mathrm{~nm}$. Samples were normalized to protein content as described above.

Non-targeted metabolomics analysis. Metabolite extracts were prepared from worms by smashing (Schütt rotation homogenizer) and heating to $70^{\circ} \mathrm{C}$ for $10 \mathrm{~min}$ in ethanol. After centrifugation the supernatant was stored at $-80^{\circ} \mathrm{C}$ until use.

Non-targeted analysis of the metabolome was performed by flow injection analysis-time of flight mass spectrometry on an Agilent 6550 QTOF instrument ${ }^{69}$ All samples were injected in duplicates. Ions were annotated based on their accurate mass and the KEGG has reference list allowing a tolerance of $0.001 \mathrm{Da}$. Unknown ions and those annotated as adducts were discarded.

Locomotion analysis. Worms were synchronized and treated for 10 days with control vector or bcat-1 RNAi as described above. After 10 days four to five worms were picked from RNAi plates and released on fresh plates without bacteria. We 
defined the refuge behaviour induced velocity as maximum speed. Directly after release $1 \mathrm{~min}$ movie clips were recorded with a Leica system (Leica M165FC with Leica camera DFC $3000 \mathrm{G}$ ). Subsequently, the videos were analysed using parallel worm tracker software developed by Goodman Lab which tracks based on centroid position of the worms ${ }^{70}$. The pixel to distance ratio was calibrated. For each treatment five independent videos were used.

Age pigment analysis. Age pigments in C. elegans reflect biological age ${ }^{26}$. Worms were synchronized and treated for 10 days with control vector or bcat-1 RNAi starting at L4 larvae stage. On day 10 worms were washed off the plates and distributed on 8 wells of a 96-well plate (Bioswisstec 96-well CG black with glass bottom, art. no.: 5241). The fluorescence of the age pigments was measured using a fluorescence plate reader (FLUOstar Omega, BMG Labtech, Offenburg, Germany; exciatition: $340-10 \mathrm{~nm}$, emission: $440-80 \mathrm{~nm}$; gain: 1,844 ). We normalized the age pigment fluorescence to the stable auto-fluorescence signal of the worms (filters: excitation: $290-10 \mathrm{~nm}$, emission: $330-10 \mathrm{~nm}$; gain: 1800) as described ${ }^{26}$.

Fertility assay. For determination of fertility we synchronized nematodes as previously described ${ }^{52}$. Single L4 larvae were transferred on single plates carrying RNAi bacteria and subsequently every $24 \mathrm{~h}$ to fresh plates. Progeny were allowed to hatch and counted. For every condition 10 worms were used.

Bcat-1 overexpression. We combined a $1 \mathrm{~kb}$ endogenous promoter with a bcat-1cDNA C-terminally fused to GFP, as well as an unc-119 rescue gene, and implemented the construct into nematodes using ballistic transformation techniques as described before ${ }^{71}$. The generated transgenic lines were selected for stable integration. The resulting strain named MIR23 carrying the integrated construct risIs[Pbcat-1::bcat-1::gfp + unc119] has been used for experiments.

Immunoblotting. Nematodes were washed three times with ice-cold S-buffer and pellets were shock-frozen in liquid nitrogen. Frozen pellets were grinded in a nitrogen-chilled mortar and suspended in phosphate buffer containing protease and phosphatase inhibitors (Complete protease inhibitor cocktail (Roche, Penzberg, Germany) and additionally $2 \mathrm{mM}$ sodium fluoride, $2 \mathrm{mM}$ sodium orthovanadate, $1 \mathrm{mM}$ phenylmethylsulphonyl fluoride and $2 \mathrm{mM}$ EDTA). Extracts were sonicated three times and centrifuged for $7 \mathrm{~min}$ at $12,000 \mathrm{~g}$ at $4{ }^{\circ} \mathrm{C}$. Supernatants were used for protein quantification, and an aliquot was boiled in Laemmli buffer and applied to SDS-polyacrylamide gel electrophoresis. Antibodies against phospho-HSF-1 (1:1,000, pSer326, Enzo, order number ADI-SPA-902-D) and alphatubulin (1:2,000, clone DM1A; Sigma-Aldrich; order number T6199) were used.

Promoter analyses. The search for transcription factor binding sites was done within the proximal bcat-1 promoter region $1 \mathrm{~kb}$ upstream of the predicted start codon. Therefore, a FASTA file containing the promoter region of bcat-1 was created using WormMart ${ }^{44}$. Next, the remaining sequence file was scanned for one or more matches to the position-specific scoring matrices of all available transcription factors using the matrix scan function of the pattern-matching programme RSAT (regulatory sequence analysis tools) $)^{72}$. The position-specific scoring matrices contains the nucleotide frequency at each position within the binding sites and were obtained from the databases Transfac and Jaspar ${ }^{73-75}$. The threshold $P$-value, which indicates the risk of false-positive predictions, was set to 0.0001 .

Statistical analyses. Data are expressed as means \pm s.d. unless otherwise indicated. Statistical analyses for all C. elegans data except lifespan assays were performed by Student's $t$-test (unpaired, two-tailed) or one-way analysis of variance (ANOVA) after testing for equal distribution of the data and equal variances within the data set. For comparing significant distributions between different groups in the lifespan assays and stress resistance assays, statistical calculations were performed using JMP software version 10 (SAS Institute Inc., Cary, NC, USA) applying the log-rank test. The comparison of correlation between bcat- 1 and $h l h-15$ expression over age was performed by applying Fisher $\mathrm{z}$ transformation of the Pearson correlation coefficients.

\section{References}

1. McCay, C. M., Crowel, M. F. \& Maynard, L. A. The effect of retarded growth upon the length of the life span and upon ultimate body size. J. Nutr. 10, 63-79 (1935).

2. Klass, M. R. A method for the isolation of longevity mutants in the nematode Caenorhabditis elegans and initial results. Mech. Ageing Dev. 22, 279-286 (1983).

3. Kirkwood, T. B. \& Melov, S. On the programmed/non-programmed nature of ageing within the life history. Curr. Biol. 21, R701-R707 (2011).

4. Gems, D. What is an anti-aging treatment? Exp. Gerontol. 58C, 14-18 (2014).

5. Johnson, T. E. Increased life-span of age-1 mutants in Caenorhabditis elegans and lower Gompertz rate of aging. Science 249, 908-912 (1990).

6. Kenyon, C., Chang, J., Gensch, E., Rudner, A. \& Tabtiang, R. A C. elegans mutant that lives twice as long as wild type. Nature 366, 461-464 (1993).
7. Tatar, M. et al. A mutant Drosophila insulin receptor homolog that extends life-span and impairs neuroendocrine function. Science 292, 107-110 (2001).

8. Clancy, D. J. et al. Extension of life-span by loss of CHICO, a Drosophila insulin receptor substrate protein. Science 292, 104-106 (2001).

9. Brown-Borg, H. M., Borg, K. E., Meliska, C. J. \& Bartke, A. Dwarf mice and the ageing process. Nature 384, 33 (1996).

10. Holzenberger, M. et al. IGF-1 receptor regulates lifespan and resistance to oxidative stress in mice. Nature 421, 182-187 (2003).

11. Blüher, M., Kahn, B. B. \& Kahn, C. R. Extended longevity in mice lacking the insulin receptor in adipose tissue. Science 299, 572-574 (2003).

12. Bonafe, M. et al. Polymorphic variants of insulin-like growth factor I (IGF-I) receptor and phosphoinositide 3-kinase genes affect IGF-I plasma levels and human longevity: cues for an evolutionarily conserved mechanism of life span control. J. Clin. Endocrinol. Metab. 88, 3299-3304 (2003).

13. van Heemst, D. et al. Reduced insulin/IGF-1 signalling and human longevity. Aging Cell 4, 79-85 (2005).

14. Pawlikowska, L. et al. Association of common genetic variation in the insulin/IGF1 signaling pathway with human longevity. Aging Cell 8, 460-472 (2009).

15. Burkewitz, K., Zhang, Y. \& Mair, W. B. AMPK at the nexus of energetics and aging. Cell Metab. 20, 10-25 (2014).

16. Martinez de Morentin, P. B. et al. Hypothalamic mTOR: the rookie energy sensor. Curr. Mol. Med. 14, 3-21 (2014).

17. Johnson, S. C., Rabinovitch, P. S. \& Kaeberlein, M. mTOR is a key modulator of ageing and age-related disease. Nature 493, 338-345 (2013).

18. Sinclair, D. \& Verdin, E. The longevity of sirtuins. Cell Rep. 2, 1473-1474 (2012).

19. Sena, L. A. \& Chandel, N. S. Physiological roles of mitochondrial reactive oxygen species. Mol. Cell 48, 158-167 (2012).

20. Shadel, G. S. \& Horvath, T. L. Mitochondrial ROS signaling in organismal homeostasis. Cell 163, 560-569 (2015).

21. Lee, S. S. et al. A systematic RNAi screen identifies a critical role for mitochondria in C. elegans longevity. Nat. Genet. 33, 40-48 (2003).

22. Hamilton, B. et al. A systematic RNAi screen for longevity genes in C. elegans Genes Dev. 19, 1544-1555 (2005).

23. McCarroll, S. A. et al. Comparing genomic expression patterns across species identifies shared transcriptional profile in aging. Nat. Genet. 36, 197-204 (2004).

24. McCormick, M. A. et al. A comprehensive analysis of replicative lifespan in 4,698 single-gene deletion strains uncovers conserved mechanisms of aging. Cell Metab 22, 895-906 (2015).

25. Fuchs, S. et al. A metabolic signature of long life in Caenorhabditis elegans. BMC Biol. 8, 14 (2010).

26. Gerstbrein, B., Stamatas, G., Kollias, N. \& Driscoll, M. In vivo spectrofluorimetry reveals endogenous biomarkers that report healthspan and dietary restriction in Caenorhabditis elegans. Aging Cell 4, 127-137 (2005).

27. Robida-Stubbs, S. et al. TOR signaling and rapamycin influence longevity by regulating SKN-1/Nrf and DAF-16/FoxO. Cell Metab. 15, 713-724 (2012).

28. Calixto, A., Chelur, D., Topalidou, I., Chen, X. \& Chalfie, M. Enhanced neuronal RNAi in C. elegans using SID-1. Nat. Methods 7, 554-559 (2010).

29. Cota, D. et al. Hypothalamic mTOR signaling regulates food intake. Science 312, 927-930 (2006).

30. Schackwitz, W. S., Inoue, T. \& Thomas, J. H. Chemosensory neurons function in parallel to mediate a pheromone response in C. elegans. Neuron 17, 719-728 (1996).

31. Ogg, S. et al. The Fork head transcription factor DAF-16 transduces insulin-like metabolic and longevity signals in C. elegans. Nature 389, 994-999 (1997).

32. Shaw, W. M., Luo, S., Landis, J., Ashraf, J. \& Murphy, C. T. The C. elegans TGF-beta dauer pathway regulates longevity via insulin signaling. Curr. Biol. 17, 1635-1645 (2007).

33. Yan, J. et al. Obesity- and aging-induced excess of central transforming growth factor-beta potentiates diabetic development via an RNA stress response. Nat Med. 20, 1001-1008 (2014).

34. Tonjes, M. et al. BCAT1 promotes cell proliferation through amino acid catabolism in gliomas carrying wild-type IDH1. Nat. Med. 19, 901-908 (2013).

35. Lang, C. H., Lynch, C. J. \& Vary, T. C. BCATm deficiency ameliorates endotoxin-induced decrease in muscle protein synthesis and improves survival in septic mice. Am. J. Physiol. Regul. Integr. Comp. Physiol. 299, R935-R944 (2010).

36. Greco, D. et al. Gene expression in human NAFLD. Am. J. Physiol. Gastrointest. Liver. Physiol. 294, G1281-G1287 (2008).

37. Angulo, P. Nonalcoholic fatty liver disease. N. Engl. J. Med. 346, 1221-1231 (2002).

38. Hall, T. R., Wallin, R., Reinhart, G. D. \& Hutson, S. M. Branched chain aminotransferase isoenzymes. Purification and characterization of the rat brain isoenzyme. J. Biol. Chem. 268, 3092-3098 (1993). 
39. She, P. et al. Disruption of BCATm in mice leads to increased energy expenditure associated with the activation of a futile protein turnover cycle. Cell Metab. 6, 181-194 (2007).

40. Shin, A. C. et al. Brain insulin lowers circulating BCAA levels by inducing hepatic BCAA catabolism. Cell Metab. 20, 898-909 (2014).

41. Newgard, C. B. et al. A branched-chain amino acid-related metabolic signature that differentiates obese and lean humans and contributes to insulin resistance. Cell Metab. 9, 311-326 (2009).

42. Bishop, N. A. \& Guarente, L. Two neurons mediate diet-restriction-induced longevity in C. elegans. Nature 447, 545-549 (2007).

43. D'Antona, G. et al. Branched-chain amino acid supplementation promotes survival and supports cardiac and skeletal muscle mitochondrial biogenesis in middle-aged mice. Cell Metab. 12, 362-372 (2010).

44. Harris, T. W. et al. WormBase 2014: new views of curated biology. Nucleic Acids Res. 42, D789-D793 (2014).

45. Zhang, G. et al. Hypothalamic programming of systemic ageing involving IKKbeta, NF-kappaB and GnRH. Nature 497, 211-216 (2013).

46. Satoh, A. et al. Sirtl extends life span and delays aging in mice through the regulation of Nk2 homeobox 1 in the DMH and LH. Cell Metab. 18, 416-430 (2013).

47. Chou, S. D., Prince, T., Gong, J. \& Calderwood, S. K. mTOR is essential for the proteotoxic stress response, HSF1 activation and heat shock protein synthesis. PLoS One 7, e39679 (2012)

48. Barna, J. et al. Heat shock factor-1 intertwines insulin/IGF-1, TGF-beta and cGMP signaling to control development and aging. BMC Dev. Biol. 12, 32 (2012).

49. Gumienny, T. L. \& Savage-Dunn, C. TGF- $\beta$ signaling in C. elegans (2013), online only http://www.wormbook.org/chapters/www_tgfbsignal/ tgfbsignal.html.

50. Brenner, S. The genetics of Caenorhabditis elegans. Genetics 77, 71-94 (1974).

51. Zarse, K. et al. Impaired insulin/IGF1-signaling extends life span by promoting mitochondrial L-proline catabolism to induce a transient ROS signal. Cell Metab. 15, 451-465 (2012).

52. Schulz, T. J. et al. Glucose restriction extends Caenorhabditis elegans life span by inducing mitochondrial respiration and increasing oxidative stress. Cell Metab. 6, 280-293 (2007).

53. Langmead, B., Trapnell, C., Pop, M. \& Salzberg, S. L. Ultrafast and memory efficient alignment of short DNA sequences to the human genome. Genome Biol. 10, R25 (2009).

54. Mortazavi, A., Williams, B. A., McCue, K., Schaeffer, L. \& Wold, B. Mapping and quantifying mammalian transcriptomes by RNA-Seq. Nat. Methods 5, 621-628 (2008).

55. Anders, S. \& Huber, W. Differential expression analysis for sequence count data. Genome Biol. 11, R106 (2010).

56. Robinson, M. D., McCarthy, D. J. \& Smyth, G. K. edgeR: a Bioconductor package for differential expression analysis of digital gene expression data. Bioinformatics 26, 139-140 (2010).

57. Hardcastle, T. \& Kelly, K. baySeq: Empirical Bayesian methods for identifying differential expression in sequence count data. BMC Bioinformatics 11, 422 (2010).

58. Priebe, S. \& Menzel, U. in Proceedings of the International Conference on Bioinformatics Models, Methods and Algorithms. Vol. 1 (eds Gamboa, H., Fred, ALN,, Solé-Casals, J. \& Fernandes, P.) 105-110 (Barcelona: Scitepress - Science and Technology Publications, 2013).

59. Cannon, R. L., Dave, J. V. \& Bezdek, J. C. Efficient implementation of the fuzzy c-means clustering algorithms. IEEE Trans. Pattern Anal. Mach. Intell. 8, 248-255 (1986).

60. Guthke, R., Möller, U., Hoffmann, M., Thies, F. \& Töpfer, S. Dynamic network reconstruction from gene expression data applied to immune response during bacterial infection. Bioinformatics 21, 1626-1634 (2005).

61. Karp, P. D. et al. Pathway Tools version 13.0: integrated software for pathway/genome informatics and systems biology. Brief Bioinform. 11, 40-79 (2010).

62. Kim, D. et al. TopHat2: accurate alignment of transcriptomes in the presence of insertions, deletions and gene fusions. Genome Biol. 14, R36 (2013).

63. Liao, Y., Smyth, G. K. \& Shi, W. featureCounts: an efficient general purpose program for assigning sequence reads to genomic features. Bioinformatics 30, 923-930 (2014).

64. Luo, W., Friedman, M., Shedden, K., Hankenson, K. \& Woolf, P. GAGE: generally applicable gene set enrichment for pathway analysis. $B M C$ Bioinformatics 10, 161 (2009).

65. Pfaffl, M. W., Horgan, G. W. \& Dempfle, L. Relative expression software tool (REST C) for group-wise comparison and statistical analysis of relative expression results in real-time PCR. Nucleic Acids Res. 30, e36 (2002).

66. Schmeisser, S. et al. Neuronal ROS signaling rather than AMPK/sirtuinmediated energy sensing links dietary restriction to lifespan extension. Mol. Metab. 2, 92-102 (2013).
67. Bradford, M. M. A rapid and sensitive method for the quantitation of microgram quantities of protein utilizing the principle of protein-dye binding. Anal. Biochem. 72, 248-254 (1976).

68. Smith, P. K. et al. Measurement of protein using bicinchoninic acid. Anal. Biochem. 150, 76-85 (1985).

69. Fuhrer, T., Heer, D., Begemann, B. \& Zamboni, N. High-throughput, accurate mass metabolome profiling of cellular extracts by flow injection-time-of-flight mass spectrometry. Anal. Chem. 83, 7074-7080 (2011).

70. Ramot, D., Johnson, B. E., Berry, Jr T. L., Carnell, L. \& Goodman, M. B. The Parallel Worm Tracker: a platform for measuring average speed and druginduced paralysis in nematodes. PLoS ONE 3, e2208 (2008).

71. Schmeisser, K. et al. Role of sirtuins in lifespan regulation is linked to methylation of nicotinamide. Nat. Chem. Biol. 9, 693-700 (2013).

72. Turatsinze, J. V., Thomas-Chollier, M., Defrance, M. \& van Helden, J. Using RSAT to scan genome sequences for transcription factor binding sites and cisregulatory modules. Nat. Protoc. 3, 1578-1588 (2008).

73. Matys, V. et al. TRANSFAC: transcriptional regulation, from patterns to profiles. Nucleic Acids Res. 31, 374-378 (2003).

74. Sandelin, A., Alkema, W., Engstrom, P., Wasserman, W. W. \& Lenhard, B. JASPAR: an open-access database for eukaryotic transcription factor binding profiles. Nucleic Acids Res. 32, D91-D94 (2004).

75. Mathelier, A. et al. JASPAR 2014: an extensively expanded and updated open-access database of transcription factor binding profiles. Nucleic Acids Res. 42, D142-D147 (2014).

\section{Acknowledgements}

C. elegans strains used in this work were provided by the Caenorhabditis Genetics Centre (University of Minnesota, USA), which is funded by NIH Office of Research Infrastructure Programs (P40 OD010440). The excellent technical assistance of Madlen Guenther, Ivonne Heinze, Beate Laube, Annett Müller, Susann Richter and Waltraud Scheiding, the excellent secretarial assistance of Marina Molinari are gratefully acknowledged. This work was supported by the research programme of the Jena Centre for Systems Biology of Aging (JenAge) funded by the German Ministry for Education and Research (Bundesministerium für Bildung und Forschung/BMBF 0315581), the German Research Association (Deutsche Forschungsgemeinschaft, DFG) Research Training Group 1715 Molecular Signatures of Adaptive Stress Responses, Jena, Germany, and the Swiss National Science Foundation (Schweizerischer Nationalfonds, SNF 31003A_156031).

\section{Author contributions}

J.M. performed all experiments except stated otherwise. N.U. and K.Z. performed lifespan analyses depicted in Figs 3-6 and table 1. S.P., J.S. and R.G. analysed RNA-seq data. M.G. and M.P. performed RNA-seq analyses. C.F. and O.W.W. obtained M. musculus ageing samples. N.H. and C.E. obtained D. rerio samples. S.S. and A.D. obtained C. elegans ageing samples. S.M. and P.H. performed laser ablation in nematodes. S.B.S. and M.K. performed amino acid quantifications. N.Z. performed metabolomics analyses. J.G. and C.K. performed in silico pathway analyses. Me.R. and D.K. performed qPCR analyses. Me.R. performed locomotion analyses. J.S., Mi.R., R.G. C.K. M.P., K.Z., C.E., O.W.W. and P.H. designed the initial part of the study (Figs 1 and 2), J.M., K.Z. and Mi.R. designed the subsequent parts. J.M. and Mi.R. wrote the paper.

\section{Additional information}

Accession codes: RNA-Seq data were deposited in gene expression omnibus (GEO) under accession codes GSE46051 (normal ageing of C. elegans), GSE46916 (normal ageing of D. rerio and M. musculus) and GSE60672 (bcat-1 RNAi perturbation in C. elegans).

Supplementary Information accompanies this paper at http://www.nature.com/ naturecommunications

Competing financial interests: The authors declare no competing financial interests.

Reprints and permission information is available online at http://npg.nature.com/ reprintsandpermissions/

How to cite this article: Mansfeld, J. et al. Branched-chain amino acid catabolism is a conserved regulator of physiological ageing. Nat. Commun. 6:10043 doi: 10.1038/ ncomms10043 (2015)

This work is licensed under a Creative Commons Attribution 4.0 International License. The images or other third party material in this article are included in the article's Creative Commons license, unless indicated otherwise in the credit line; if the material is not included under the Creative Commons license, users will need to obtain permission from the license holder to reproduce the material. To view a copy of this license, visit http://creativecommons.org/licenses/by/4.0/ 